PROCEEDINGS OF THE

AMERICAN MATHEMATICAL SOCIETY

Volume 139, Number 5, May 2011, Pages 1605-1615

S 0002-9939(2011)10823-2

Article electronically published on January 11, 2011

\title{
CARLESON MEASURES ON DIRICHLET-TYPE SPACES
}

\author{
GERARDO R. CHACÓN
}

(Communicated by Richard Rochberg)

\begin{abstract}
We show that a maximal inequality holds for the non-tangential maximal operator on Dirichlet spaces with harmonic weights on the open unit disc. We then investigate two notions of Carleson measures on these spaces and use the maximal inequality to give characterizations of the Carleson measures in terms of an associated capacity.
\end{abstract}

\section{INTRODUCTION}

Given a positive Borel measure $\mu$ defined on the boundary of the unit disc $\partial \mathbb{D}$, let $P_{\mu}$ be positive the harmonic function defined on the unit disc $\mathbb{D}$ by

$$
P_{\mu}(z)=\int_{0}^{2 \pi} \frac{1-|z|^{2}}{\left|e^{i t}-z\right|^{2}} \frac{d \mu(t)}{2 \pi} .
$$

Definition 1.1. The harmonic Dirichlet space $\mathcal{B}_{\mu}$ consists of all real functions $f \in L^{2}(\partial \mathbb{D})$ such that

$$
\|f\|_{\mathcal{B}_{\mu}}^{2}:=\|f\|_{L^{2}(\partial \mathbb{D})}^{2}+\int_{\mathbb{D}}|\nabla(f)|^{2} P_{\mu} d A<\infty,
$$

where $\nabla(f)$ denotes the gradient of the harmonic extension of $f$ to $\mathbb{D}$ and $d A$ denotes the normalized Lebesgue area measure on $\mathbb{D}$.

The Dirichlet type space $D(\mu)$ is defined as the space of all analytic functions on $\mathbb{D}$ such that

$$
\int_{\mathbb{D}}\left|f^{\prime}(z)\right|^{2} P_{\mu}(z) d A(z)<\infty .
$$

If $\mu=0$, then define $D(\mu)=H^{2}$, the Hardy space on the unit disc. Notice that if $d \mu=d m$ is the arc-length Lebesgue measure on $\partial \mathbb{D}$, then the Dirichlet-type space $D(m)$ coincides with the classical Dirichlet space $\mathcal{D}$.

Dirichlet-type spaces were introduced by Richter in [6] when he was investigating analytic two-isometries. Richter showed that every analytic two-isometry $T$ such that $\operatorname{dim} \operatorname{ker} T^{*}=1$ can be represented as muliplication by $z$ on a Dirichlet-type space $D(\mu)$. These spaces have been studied ever since by several authors; see for example [2], 3, 4], 8], 10, 11, 12, 14] and [13.

Received by the editors April 26, 2010.

2000 Mathematics Subject Classification. Primary 42B25; Secondary 46C99, 31C25.

Key words and phrases. Dirichlet-type spaces, non-tangential maximal function, maximal inequality, Carleson measures.

(C)2011 American Mathematical Society Reverts to public domain 28 years from publication 
It is shown in [6] that the space $D(\mu)$ is contained as a set in the space $H^{2}$. Consequently a norm on $D(\mu)$ can be defined as

$$
\|f\|_{D(\mu)}^{2}:=\|f\|_{H^{2}}^{2}+\int_{\mathbb{D}}\left|f^{\prime}(z)\right|^{2} P_{\mu}(z), d A(z),
$$

and it can be shown that evaluation functionals are continuous on $D(\mu)$.

Definition 1.2. Given a function $f$ on $\mathbb{D}$, the non-tangential maximal function of $f$ is the function on $\partial \mathbb{D}$ defined by

$$
N(f)\left(e^{i \theta}\right):=\sup _{z \in \Gamma\left(e^{i \theta}\right)}|f(z)|
$$

where $\Gamma\left(e^{i \theta}\right)$ denotes the convex hull of the disk $\{|z|<1 / 2\}$ and the point $e^{i \theta}$.

In [3] and [4], Chartrand investigates some properties of Carleson measures on Dirichlet-type spaces and proves a maximal-type inequality for the case in which $\mu$ is a finitely atomic measure and in the case in which $\mu=w d \sigma$, where $\sigma$ denotes the arc-length measure and $w$ is a Muckenhoupt weight. Here we show that a maximal inequality holds for the general case. This is our main theorem.

Theorem 1.3. Let $\mu$ be a finite, positive Borel measure on $\partial \mathbb{D}$. Then there exists a constant $C>0$ such that for every $f \in D(\mu)$,

$$
\|N f\|_{D(\mu)} \leq C\|f\|_{D(\mu)} .
$$

Once we have proved the maximal inequality, we can follow Stegenga's approach 15 and use appropriate capacities to characterize the Carleson measures for the $D(\mu)$ spaces (Theorem 1.5 ).

We will say that a positive finite Borel measure $\nu$ is a Carleson measure for the Dirichlet space $D(\mu)$ if there exists a constant $C>0$ such that for every function $f \in D(\mu)$ the following inequality holds:

$$
\int|f|^{2} d \nu \leq C\|f\|_{D(\mu)}^{2}
$$

Definition 1.4. For any open set $O \subset \partial \mathbb{D}$ define the $\mathcal{B}_{\mu}$-capacity of $O$ by

$$
\operatorname{cap}_{\mathcal{B}_{\mu}}(O):=\inf \left\{\|f\|_{\mathcal{B}_{\mu}}^{2}: f \geq 1 \text { a.e. on } O\right\} \text {. }
$$

Theorem 1.5. Let $\mu$ be a finite, positive Borel measure on $\partial \mathbb{D}$. Then a positive Borel measure $\nu$ is a $D(\mu)$-Carleson measure if and only if there exists a constant $C>0$ such that for any open set $O \subset \partial \mathbb{D}$

$$
\nu(T(O)) \leq C \operatorname{cap}_{\mathcal{B}_{\mu}}(O),
$$

where $T(O):=\left\{z \in \mathbb{D}:\left\{e^{i \theta}:\left|e^{i \theta}-z /\right| z||<1-|z|\right\} \subset O\right\}$.

\section{The nOn-tangential maximal FUnCtion on $D(\mu)$}

In this section, we will show an inequality for the local Dirichlet integral of the non-tangential maximal function of a function in $D(\mu)$. First, we will reduce the problem to one of the harmonic functions by using techniques that can be found in [16] in which the case of the Dirichlet spaces $D_{\alpha}^{p}$ is considered.

In [7], Richter and Sundberg introduced the notion of a Local Dirichlet Integral: Let $f \in L^{1}(\partial \mathbb{D})$ and $\zeta \in \partial \mathbb{D}$. We assume that $f\left(e^{i t}\right)$ equals the non-tangential limit 
of its Poisson extension whenever the latter exists. Let $\zeta \in \mathbb{D}$; the local Dirichlet integral of $f$ at $\zeta$ is given by

$$
D_{\zeta}(f)=\int_{0}^{2 \pi}\left|\frac{f\left(e^{i t} \zeta\right)-f(\zeta)}{e^{i t}-1}\right|^{2} \frac{d t}{2 \pi} .
$$

If $f(\zeta)$ does not exist, then we set $D_{\zeta}(f)=\infty$.

Then the norm of the Dirichlet-type space $D(\mu)$ is given by

$$
\|f\|_{D(\mu)}^{2}:=\|f\|_{H^{2}}+\int D_{\zeta}(f) d \mu(\zeta)<\infty .
$$

We will show that for every $f \in D(\mu)$ we have that $\|f\|_{D(\mu)} \sim\|\operatorname{Re} f\|_{\mathcal{B}_{\mu}}$, and consequently it will be enough to prove a maximal inequality for harmonic functions. For that, we will use the following proposition.

Proposition 2.1 ([3, Prop. 2.9]). Let $f$ be a harmonic function on $\mathbb{D}$ of the form $f=f_{+}+f_{-}$, where $f_{+}, f_{-} \in D(\mu)$, and $f_{-}(0)=0$. Then

$$
\int_{\partial \mathbb{D}} D_{\zeta}(f) d \mu(\zeta)=\int_{\mathbb{D}}|\nabla(f)|^{2} P_{\mu} d A .
$$

Now, with this in hand, we have that if $f \in D(\mu)$, then there exist harmonic functions $h_{1}$ and $h_{2}$ such that $f=h_{1}+i h_{2}$ and $h_{1}$ satisfies the conditions of the previous proposition. Moreover, since $h_{1}$ and $h_{2}$ are harmonic conjugates, then (see [5], Theorem 4.1) $\left\|h_{2}\right\|_{L^{2}(\partial \mathbb{D})} \lesssim\left\|h_{1}\right\|_{L^{2}(\partial \mathbb{D})}$. Consequently,

$$
\begin{aligned}
\|f\|_{D(\mu)}^{2} & =\|f\|_{H^{2}}^{2}+\int_{\mathbb{D}}\left|f^{\prime}\right|^{2} P_{\mu} d A \\
& \lesssim\left\|h_{1}\right\|_{L^{2}(\mu)}^{2}+\left\|h_{2}\right\|_{L^{2}(\mu)}^{2}+\int_{\mathbb{D}}\left|\nabla h_{1}\right|^{2} P_{\mu} d A \\
& \lesssim\left\|h_{1}\right\|_{L^{2}(\mu)}^{2}+\int_{\mathbb{D}}\left|\nabla h_{1}\right|^{2} P_{\mu} d A \\
& =\left\|h_{1}\right\|_{\mathcal{B}_{\mu}}^{2} .
\end{aligned}
$$

On the other hand, it is clear that $\|h\|_{\mathcal{B}_{\mu}} \lesssim\|f\|_{D(\mu)}$. Consequently, we have that

$$
\|f\|_{D(\mu)} \sim\|\operatorname{Ref}\|_{\mathcal{B}_{\mu}} .
$$

Now, we will use a truncation method to show the maximal inequality for functions in $\mathcal{B}_{\mu}$ and consequently in $D(\mu)$.

Let $\varphi$ be a non-decreasing function in $C_{0}^{\infty}(\mathbb{R})$ which satisfies

$$
\varphi(t)= \begin{cases}0, & \text { if } t \leq 1 / 2 \\ 1, & \text { if } t \geq 1\end{cases}
$$

and consider the smooth truncation $\left\{F_{j}\right\}_{-\infty}^{\infty}$ :

$$
F_{j}(f):=2^{j} \varphi\left(\frac{|f|}{2^{j}}\right), \quad j=0, \pm 1, \pm 2, \ldots
$$


Then for each $j$ we have that

$$
\begin{aligned}
\left\|F_{j}(f)\right\|_{L^{2}(\partial \mathbb{D})}^{2} & =2^{2 j} \int_{\partial \mathbb{D}}\left|\varphi\left(\frac{\left|f\left(e^{i t}\right)\right|}{2^{j}}\right)\right|^{2} \frac{d t}{2 \pi} \\
& =2^{2 j} \int_{\left\{|f|>2^{j-1}\right\}}\left|\varphi\left(\frac{\left|f\left(e^{i t}\right)\right|}{2^{j}}\right)\right|^{2} \frac{d t}{2 \pi} \\
& \leq 2^{2 j}\left|\left\{|f|>2^{j-1}\right\}\right|,
\end{aligned}
$$

where for a set $A \subset \partial \mathbb{D},|A|$ denotes the normalized Lebesgue measure of $A$ on $\partial \mathbb{D}$. Consequently,

$$
\begin{aligned}
\sum_{j=-\infty}^{\infty}\left\|F_{j}(f)\right\|_{L^{2}(\partial \mathbb{D})}^{2} & \leq \sum_{j=-\infty}^{\infty} 2^{2 j}\left|\left\{|f|>2^{j-1}\right\}\right| \\
& \lesssim \sum_{j=-\infty}^{\infty} \int_{2^{j-1}}^{2^{j}} 2^{j-1}\left|\left\{|f|>2^{j-1}\right\}\right| d t \\
& \lesssim \sum_{j=-\infty}^{\infty} \int_{2^{j-1}}^{2^{j}}|\{|f|>t\}| t d t \\
& =\int_{0}^{\infty}|\{|f|>t\}| t d t \\
& =\|f\|_{L^{2}(\partial \mathbb{D})}^{2} .
\end{aligned}
$$

Lemma 2.2. There exists a constant $C>0$ such that

$$
\sum_{j=-\infty}^{\infty}\left\|F_{j}(f)\right\|_{\mathcal{B}_{\mu}}^{2} \leq C\|f\|_{\mathcal{B}_{\mu}}^{2}
$$

Proof. The corresponding inequality for the $L^{2}(\partial \mathbb{D})$-norm is shown above. Thus, it is enough to show that there exists a constant $C>0$ such that for any $t, s \in \mathbb{R}$,

$$
\sum_{l=-\infty}^{\infty}\left|\frac{F_{l}\left(f\left(e^{i t}\right)\right)-F_{l}\left(f\left(e^{i s}\right)\right)}{e^{i t}-e^{i s}}\right|^{2} \leq\left|\frac{f\left(e^{i t}\right)-f\left(e^{i s}\right)}{e^{i t}-e^{i s}}\right|^{2}
$$

The proof of this is done in [16]. We include it here for the sake of completeness. Let $j$ and $k$ be integers such that $2^{j-1} \leq\left|f\left(e^{i t}\right)\right|<2^{j}$ and $2^{k-1} \leq\left|f\left(e^{i t}\right)\right|<2^{k}$. Without loss of generality we may assume that $j \geq k$. Then for the case $j=k$ we use the Mean Value Theorem for the function $\varphi$ to obtain that there exists a constant $c \in(0,1)$ such that

$$
\begin{aligned}
\sum_{l=-\infty}^{\infty}\left|F_{l}\left(f\left(e^{i t}\right)\right)-F_{l}\left(f\left(e^{i s}\right)\right)\right|^{2} & =\left|F_{j}\left(f\left(e^{i t}\right)\right)-F_{j}\left(f\left(e^{i s}\right)\right)\right|^{2} \\
& =\left.\left|\varphi^{\prime}(c)\right|^{2}|| f\left(e^{i t}\right)|-| f\left(e^{i s}\right)\right|^{2} \\
& \lesssim\left|f\left(e^{i t}\right)-f\left(e^{i s}\right)\right|^{2}
\end{aligned}
$$

and inequality (2.2) holds. 
Now, if $j \geq k+1$, then we use the Mean Value Theorem twice to find two constants $c$ and $d$ in $(0,1)$ such that

$$
\begin{aligned}
\sum_{l=-\infty}^{\infty}\left|F_{l}\left(f\left(e^{i t}\right)\right)-F_{l}\left(f\left(e^{i s}\right)\right)\right|^{2}= & \left|2^{k}-F_{k}\left(f\left(e^{i s}\right)\right)\right|^{2}+\left|F_{j}\left(f\left(e^{i t}\right)\right)\right|^{2} \\
= & 2^{2 k}\left|\varphi(1)-\varphi\left(2^{-k} \mid f\left(e^{i s} \mid\right)\right)\right|^{2} \\
& +2^{2 j}\left|\varphi\left(2^{-j}\left|f\left(e^{i t}\right)\right|\right)-\varphi(1 / 2)\right|^{2} \\
= & \left|\varphi^{\prime}(c)\right|^{2}\left(2^{k}-\left|f\left(e^{i s}\right)\right|\right)^{2} \\
& +\left|\varphi^{\prime}(d)\right|^{2}\left(\left|f\left(e^{i t}\right)\right|-2^{j-1}\right)^{2} \\
\lesssim & \left(\left|f\left(e^{i t}\right)-f\left(e^{i s}\right)\right|\right)^{2} \lesssim\left|f\left(e^{i t}\right)-f\left(e^{i s}\right)\right|^{2}
\end{aligned}
$$

and inequality (2.2) also holds.

Lemma 2.3. For all $f \in \mathcal{B}_{\mu}$ we have the estimate

$$
\int_{0}^{\infty} \operatorname{cap}_{\mathcal{B}_{\mu}}(\{|f|>t\}) t d t \lesssim\|f\|_{\mathcal{B}_{\mu}}^{2} .
$$

Proof. Since

$$
\begin{aligned}
\int_{0}^{\infty} \operatorname{cap}_{\mathcal{B}_{\mu}}(\{|f|>t\}) t d t & =\sum_{j=-\infty}^{\infty} \int_{2^{j}}^{2^{j+1}} \operatorname{cap}_{\mathcal{B}_{\mu}}(\{|f|>t\}) t d t \\
& \leq \sum_{j=-\infty}^{\infty} \int_{2^{j}}^{2^{j+1}} 2^{j} \operatorname{cap}_{\mathcal{B}_{\mu}}\left(\left\{|f|>2^{j}\right\}\right) d t \\
& =\sum_{j=-\infty}^{\infty} 2^{2 j} \operatorname{cap}_{\mathcal{B}_{\mu}}\left(\left\{|f|>2^{j}\right\}\right)
\end{aligned}
$$

and $2^{-k} F_{k}(f) \geq 1$ on the set $\left\{|f|>2^{k}\right\}$, then using Lemma 2.2 we have that

$$
\sum_{-\infty}^{\infty} 2^{2 k} \operatorname{cap}_{\mathcal{B}_{\mu}}\left(\left\{|f|>2^{k}\right\}\right) \leq \sum_{-\infty}^{\infty}\left\|F_{k}(f)\right\|_{\mathcal{B}_{\mu}}^{2} \lesssim\|f\|_{\mathcal{B}_{\mu}}^{2} .
$$

We will show that the operator $N$ satisfies

$$
D_{\zeta}(N f) \lesssim D_{\zeta}(f) .
$$

For a function $g \in L^{1}(\partial \mathbb{D})$ define the following function as

$$
M g\left(e^{i x}\right):=\sup _{1 \in I} \frac{1}{|I|} \int_{I} \frac{\left|e^{i t}-1\right|\left|g\left(e^{i(x+t)}\right)-g\left(e^{i t}\right)\right|}{\left|e^{i x}-1\right|} \frac{d t}{2 \pi},
$$

where the supremum is taken over all the open intervals $I \subset \partial \mathbb{D}$ centered at 1 . It is well known (see for example 9 ) that for every $e^{i x} \in \partial \mathbb{D}$,

$$
\sup _{z \in \Gamma(1)}\left|g\left(z e^{i x}\right)-g(z)\right| \lesssim \sup _{1 \in I} \frac{1}{|I|} \int_{I}\left|g\left(e^{i(x+t)}\right)-g\left(e^{i t}\right)\right| \frac{d t}{2 \pi} .
$$

We will also need the following lemmas.

Lemma 2.4. Let $g \in L^{1}(\partial \mathbb{D})$. Then for every $\lambda>0$

$$
\left|\left\{e^{i x} \in \partial \mathbb{D}: M g\left(e^{i x}\right)>\lambda\right\}\right| \lesssim \frac{\|g\|_{L^{1}(\partial \mathbb{D})}}{\lambda} ;
$$

i.e. $M$ maps $L^{1}(\partial \mathbb{D})$ to weak- $L^{1}(\partial \mathbb{D})$. 
Proof. Notice that

$$
\begin{aligned}
M g\left(e^{i x}\right) & \leq \sup _{1 \in I} \int_{I} \frac{\left|g\left(e^{i(x+t)}\right)-g\left(e^{i t}\right)\right|}{\left|e^{i x}-1\right|} \frac{d t}{2 \pi} \\
& \lesssim \frac{1}{\left|e^{i x}-1\right|}\|g\|_{L^{1}(\partial \mathbb{D})} .
\end{aligned}
$$

Consequently,

$$
\left\{e^{i x} \in \partial \mathbb{D}: M g\left(e^{i x}\right)>\lambda\right\} \subset\left\{e^{i x} \in \partial \mathbb{D}: \frac{1}{\left|e^{i x}-1\right|}\|g\|_{L^{1}(\partial \mathbb{D})}>\lambda\right\},
$$

and the result follows.

By equation (2.3) we have that

$$
\sup _{z \in \Gamma(1)}|z-1| \frac{\left|g\left(z e^{i x}\right)-g(z)\right|}{\left|e^{i x}-1\right|} \lesssim M g\left(e^{i x}\right),
$$

which, by the previous lemma, implies that the operator defined as

$$
\widetilde{M} g\left(e^{i x}\right):=\sup _{z \in \Gamma(1)}|z-1| \frac{\left|g\left(z e^{i x}\right)-g(z)\right|}{\left|e^{i x}-1\right|}
$$

maps $L^{1}(\partial \mathbb{D})$ to weak- $L^{1}(\partial \mathbb{D})$.

Lemma 2.5. The sublinear operator $\widetilde{M}$ maps $L^{\infty}(\partial \mathbb{D})$ to $L^{\infty}(\partial \mathbb{D})$.

Proof. Suppose $g \in L^{\infty}(\partial \mathbb{D})$. Then

$$
\begin{aligned}
\widetilde{M} g\left(e^{i x}\right)= & \sup _{z \in \Gamma(1)}|z-1| \frac{\left|g\left(z e^{i x}\right)-g(z)\right|}{\left|e^{i x}-1\right|} \\
\sim & \sup _{0 \leq r<1} \frac{(1-r)}{\left|e^{i x}-1\right|}\left|\int_{0}^{2 \pi}\left(\frac{1-r^{2}}{\left|e^{i(t-x)}-r\right|^{2}}-\frac{1-r^{2}}{\left|e^{i t}-r\right|^{2}}\right) g\left(e^{i t}\right) \frac{d t}{2 \pi}\right| \\
\leq & \sup _{0 \leq r<1} \frac{(1-r)\left(1-r^{2}\right)}{\left|e^{i x}-1\right|} \int_{0}^{2 \pi}\left(\frac{\left|2 \operatorname{Re} r e^{-i t}\left(1-e^{i x}\right)\right|}{\left|e^{i(t-x)}-r\right|^{2}\left|e^{i t}-r\right|^{2}}\right)\left|g\left(e^{i t}\right)\right| \frac{d t}{2 \pi} \\
\lesssim & \sup _{0 \leq r<1} \frac{(1-r)\left(1-r^{2}\right)}{\left|e^{i x}-1\right|} \int_{0}^{2 \pi}\left(\frac{\left|2 \operatorname{Re}\left(r e^{-i t}-1\right)\left(1-e^{i x}\right)\right|}{\left|e^{i(t-x)}-r\right|^{2}\left|e^{i t}-r\right|^{2}}\right)\left|g\left(e^{i t}\right)\right| \frac{d t}{2 \pi} \\
& +\sup _{0 \leq r<1} \frac{(1-r)\left(1-r^{2}\right)}{\left|e^{i x}-1\right|} \int_{0}^{2 \pi}\left(\frac{\left|2 \operatorname{Re}\left(1-e^{i x}\right)\right|}{\left|e^{i(t-x)}-r\right|^{2}\left|e^{i t}-r\right|^{2}}\right)\left|g\left(e^{i t}\right)\right| \frac{d t}{2 \pi} \\
\lesssim & \sup _{0 \leq r<1} \int_{0}^{2 \pi} \frac{\left(1-r^{2}\right)^{2}}{\left|e^{i(t-x)}-r\right|^{2}\left|e^{i t}-r\right|} \frac{d t}{2 \pi}\|g\|_{L^{\infty}(\partial \mathbb{D})} \\
& +\sup _{0 \leq r<1}\left|e^{i x}-1\right| \int_{0}^{2 \pi} \frac{\left(1-r^{2}\right)^{2}}{\left|e^{i(t-x)}-r\right|^{2}\left|e^{i t}-r\right|^{2}} \frac{d t}{2 \pi}\|g\|_{L^{\infty}(\partial \mathbb{D})} \\
\lesssim & \sup _{0 \leq r<1} \int_{0}^{2 \pi} \frac{\left(1-r^{2}\right)}{\left|e^{i(t-x)}-r\right|^{2}} \frac{d t}{2 \pi}\|g\|_{L^{\infty}(\partial \mathbb{D})} \\
& +\sup _{0 \leq r<1}\left|e^{i x}-1\right| \sum_{n=-\infty}^{\infty} r^{|n|} \int_{0}^{2 \pi} e^{i n t} \frac{1-r^{2}}{\left|e^{i(t-x)}-r\right|^{2}} \frac{d t}{2 \pi}\|g\|_{L^{\infty}(\partial \mathbb{D}) .}
\end{aligned}
$$


Consequently,

$$
\begin{aligned}
\widetilde{M} g\left(e^{i x}\right) & \lesssim\|g\|_{L^{\infty}(\partial \mathbb{D})}+\left|e^{i x}-1\right|\|g\|_{L^{\infty}(\partial \mathbb{D})} \sup _{0 \leq r<1} \sum_{n=-\infty}^{\infty} r^{2|n|} e^{i n x} \\
& =\|g\|_{L^{\infty}(\partial \mathbb{D})}+\left|e^{i x}-1\right|\|g\|_{L^{\infty}(\partial \mathbb{D})} \sup _{0 \leq r<1} \frac{1-r^{2}}{\left|e^{i x}-r^{2}\right|^{2}} \\
& \lesssim\|g\|_{L^{\infty}(\partial \mathbb{D})} .
\end{aligned}
$$

Now we can use the Marcinkiewicz Interpolation Theorem (see for example 17]) to conclude that the operator $\widetilde{M}$ maps $L^{p}(\partial \mathbb{D})$ boundedly to itself for any $1<p \leq$ $\infty$. Therefore, if a function $f \in H^{1}(\mathbb{D})$ is such that

$$
D_{1}^{p}(f):=\int_{0}^{2 \pi}\left|\frac{f\left(e^{i t}\right)-f(1)}{e^{i t}-1}\right|^{p} \frac{d t}{2 \pi}<\infty
$$

then the function $g\left(e^{i t}\right):=\frac{f\left(e^{i t}\right)-f(1)}{e^{i t}-1}$ belongs to $L^{p}(\partial \mathbb{D})$ and

$$
\begin{aligned}
f\left(z e^{i x}\right)-f(z) & =\left(z e^{i x}-1\right) g\left(z e^{i x}\right)-(z-1) g(z) \\
& =z\left(e^{i x}-1\right) g\left(z e^{i x}\right)+(z-1)\left(g\left(z e^{i x}\right)-g(z)\right),
\end{aligned}
$$

and consequently,

$$
\frac{\left|f\left(z e^{i x}\right)-f(z)\right|}{\left|e^{i x}-1\right|} \leq|z|\left|g\left(z e^{i x}\right)\right|+|z-1| \frac{\left|g\left(z e^{i x}\right)-g(z)\right|}{\left|e^{i x}-1\right|} .
$$

Hence,

$$
T(f)\left(e^{i x}\right) \leq N(g)\left(e^{i x}\right)+\sup _{z \in \Gamma(1)}|z-1| \frac{\left|g\left(z e^{i x}\right)-g(z)\right|}{\left|e^{i x}-1\right|},
$$

where $T$ is defined as the sublinear operator

$$
T f\left(e^{i x}\right):=\frac{\sup _{z \in \Gamma(1)}\left|f\left(z e^{i x}\right)-f(z)\right|}{\left|e^{i x}-1\right|} .
$$

Thus,

$$
\begin{aligned}
\left(D_{1}^{p}(N f)\right)^{1 / p} & \leq\left(\int_{0}^{2 \pi}\left(T f\left(e^{i t}\right)\right)^{p} \frac{d t}{2 \pi}\right)^{1 / p} \\
& \leq\|N g\|_{L^{p}(\partial \mathbb{D})}+\|\widetilde{M} g\|_{L^{p}(\partial \mathbb{D})} \\
& \lesssim\|g\|_{L^{p}(\partial \mathbb{D})},
\end{aligned}
$$

where we have used the fact that the operator $N$ maps $L^{p}(\partial \mathbb{D})$ boundedly to itself. Therefore for any $1<p \leq \infty$,

$$
D_{1}^{p}(N f) \lesssim D_{1}^{p}(f)
$$

and notice that if $\zeta \in \partial \mathbb{D}$, for $f \in D(\mu)$ we define $g(z):=f(z \zeta)$; then $D_{\zeta}(f)=$ $D_{1}(g)$ and $D_{\zeta}(N f)=D_{1}(N g)$. Therefore, we have the more general equation

$$
D_{\zeta}(N f) \lesssim D_{\zeta}(f)
$$

where the constant involved does not depend on $\zeta$. Finally, by equation (2.5) we have that $\int_{\partial \mathbb{D}} D_{\zeta}(N f) d \mu(\zeta) \lesssim \int_{\partial \mathbb{D}} D_{\zeta}(f) d \mu(\zeta)$, and again using the fact that $\|N f\|_{H^{2}} \lesssim\|f\|_{H^{2}}$, we have proved Theorem 1.3 . 
This theorem answers a question asked by Chartrand [4] and generalizes Lemma 3.12 of [4, where the result is proven for the case in which the measure $\mu$ is absolutely continuous with respect to the Lebesgue measure and satisfies Muckenhoupt's condition.

\section{Carleson measures on $D(\mu)$ spaces}

In this section, we will characterize Carleson measures for the $D(\mu)$ spaces. In order to do that, we will rely on results from the previous section. Specifically, notice that from equation (2.1) we can conclude that a positive measure $\nu$ on $\mathbb{D}$ is a $D(\mu)$-Carleson measure if and only if there exists a constant $C>0$ such that for every $h \in \mathcal{B}_{\mu}$ we have that

$$
\int_{\mathbb{D}}|h(z)|^{2} d \nu(z) \leq C\|h\|_{\mathcal{B}_{\mu}}^{2}
$$

Proof of Theorem 1.5. Suppose $\nu$ is a $D(\mu)$-Carleson measure. By definition, there exists a function $h \in \mathcal{B}_{\mu}$ such that $h \geq 1$ on $O$ and $\|h\|_{\mathcal{B}_{\mu}}^{2} \leq 2 \operatorname{cap}_{\mathcal{B}_{\mu}}(O)$. Since $\||h|\|_{\mathcal{B}_{\mu}} \leq\|h\|_{\mathcal{B}_{\mu}}$ we can assume that $h \geq 0$ on $\partial \mathbb{D}$. Let $O=\bigcup_{j} I_{j}$, where $\left\{I_{j}\right\}$ are disjoint arcs on $\partial \mathbb{D}$. Note that $T(O)=\bigcup_{j} T\left(I_{j}\right)$. Now, since for any $z \in T\left(I_{j}\right)$ we have that $h(z) \geq \frac{1}{4 \pi}$, then

$$
\nu(T(O)) \leq(4 \pi)^{2} \int_{T(O)}|h|^{2} d \nu \leq(4 \pi)^{2} \int_{\mathbb{D}}|h|^{2} d \nu \leq C\|h\|_{\mathcal{B}_{\mu}}^{2} \leq C \operatorname{cap}_{\mathcal{B}_{\mu}}(O) .
$$

Conversely, since $\nu(\{z \in \mathbb{D}:|f(z)|>t\}) \leq \nu(T(\{N(f)>t\}))$, then by the hypothesis and the previous lemmas,

$$
\begin{aligned}
\int_{\mathbb{D}}|f(z)|^{2} d \nu & =\int_{0}^{\infty} \nu(\{z \in \mathbb{D}:|f(z)|>t\}) t d t \\
& \lesssim \int_{0}^{\infty} \operatorname{cap}_{\mathcal{B}_{\mu}}(T(\{N(f)>t\})) t d t \\
& \lesssim\|N(f)\|_{\mathcal{B}_{\mu}} \\
& \lesssim\|f\|_{\mathcal{B}_{\mu}} .
\end{aligned}
$$

In [3, Chartrand introduces the notion of a Carleson-type measure in a different way than the one used here. We will refer to that condition as condition $(\mathrm{Ch})$.

Definition 3.1 (3]). A finite, positive Borel measure $\nu$ is said to satisfy condition $(\mathrm{Ch})$ for $D(\mu)$ if there exists a constant $C>0$ such that for every $f \in D(\mu)$

$$
\int P\left(|f|^{2} \mu\right) d \nu \leq C\|f\|_{D(\mu)}^{2}
$$

where $P\left(|f|^{2} \mu\right)$ denotes the Poisson extension of the measure $|f|^{2} d \mu$ to the unit disc, i.e.

$$
P\left(|f|^{2} \mu\right)(z):=\int_{\partial \mathbb{D}} \frac{1-|z|^{2}}{|\zeta-z|^{2}}|f(\zeta)|^{2} d \mu(\zeta) .
$$

In 4] Chartrand characterizes the measures $\nu$ that satisfy condition $(\mathrm{Ch})$ for measures $\mu$ that are either a finite sum of atoms or absolutely continuous with respect to Lebesgue measure and satisfying Muckenhoupt's condition. We will show that Chartrand's definition of Carleson measures (condition $(\mathrm{Ch})$ ) and the 
definition presented in these notes are different by exhibiting two examples. In order to do that, we will need a result from [4].

Proposition 3.2. Let $\mu=\sum a_{k} \delta_{\zeta_{k}}$, a finite sum of atoms on $\partial \mathbb{D}$. Let $\nu$ be a finite, positive, Borel measure on $\mathbb{D}$. Then $\nu$ satisfies condition $(\mathrm{Ch})$ if and only if $S_{\nu}\left(\zeta_{k}\right)<\infty$ for each $k$, where $S_{\nu}(\zeta):=\int_{\mathbb{D}} \frac{1-|z|^{2}}{|\zeta-z|} d \nu(z)$.

Example 3.3. Suppose $\mu=\delta_{1}$. We will show that $\nu$ is a $D\left(\delta_{1}\right)$-Carleson measure if and only if $|z-1|^{2} d \nu$ is a Carleson measure for the Hardy space $H^{2}$.

Suppose $\nu$ is a $D\left(\delta_{1}\right)$-Carleson measure and let $g \in H^{2}$. Define $f(z):=(z-$ 1) $g(z)$. Then $f \in D\left(\delta_{1}\right)$ and

$$
\begin{aligned}
\int|z-1|^{2}|g(z)|^{2} d \nu(z) & =\int|f|^{2} d \nu \\
& \lesssim\|f\|_{H^{2}}^{2}+\|g\|_{H^{2}}^{2} \\
& \lesssim\|g\|_{H^{2}}^{2} .
\end{aligned}
$$

Hence, $|z-1|^{2} d \nu(z)$ is a Carleson measure for the Hardy space.

Conversely, suppose $|z-1|^{2} d \nu(z)$ is an $H^{2}$-Carleson measure and let $f \in D\left(\delta_{1}\right)$. Then $g \in H^{2}$, where $g(z):=\frac{f(z)-f(1)}{z-1}$ and

$$
\begin{aligned}
\int|f|^{2} d \nu & =\int|f(1)+(z-1) g(z)|^{2} d \nu \\
& \lesssim|f(1)|^{2} \nu(\mathbb{D})+\int|z-1|^{2}|g(z)|^{2} d \nu(z) \\
& \lesssim\|f\|_{D\left(\delta_{1}\right)}^{2}+\|g\|_{H^{2}}^{2} \\
& \lesssim\|f\|_{D\left(\delta_{1}\right)}^{2} .
\end{aligned}
$$

Now, for $i \in \mathbb{Z}^{+}$consider the sequences $r_{i}:=1-\frac{1}{i}$ and $a_{i}:=\frac{1}{i^{2}}$. Take $\nu=$ $\sum_{i=1}^{\infty} a_{i} \delta_{r_{i}}$. Note that $\sum a_{i}<\infty$ and consequently $\nu$ is a finite measure. Moreover, if $I \subset \partial \mathbb{D}$ is an interval such that $1 \in I$, then

$$
\begin{aligned}
\int_{S(I)}|z-1|^{2} d \nu(z) & =\sum_{r_{i}>1-|I|} a_{i}\left|r_{i}-1\right|^{2} \\
& \leq|I|^{2} \sum_{i>1 /|I|}^{\infty} \frac{1}{i^{2}} \\
& \lesssim|I| .
\end{aligned}
$$

Therefore $|z-1|^{2} d \nu(z)$ is an $H^{2}$-Carleson measure and hence $\nu$ is a $D\left(\delta_{1}\right)$-Carleson measure. However, by Proposition $3.2 \nu$ satisfies condition $(\mathrm{Ch})$ if and only if 
$\int \frac{1-|z|^{2}}{|1-z|^{2}} d \nu(z)<\infty$. However

$$
\begin{aligned}
\int \frac{1-|z|^{2}}{|1-z|^{2}} d \nu(z) & =\sum_{i=1}^{\infty} \frac{1-r_{i}^{2}}{\left(1-r_{i}\right)^{2}} a_{i} \\
& =\sum_{i=1}^{\infty} \frac{1+r_{i}}{\left(1-r_{i}\right)} a_{i} \\
& \gtrsim \sum_{i=1}^{\infty} \frac{1}{i}=\infty .
\end{aligned}
$$

So, $\nu$ is a $D\left(\delta_{1}\right)$-Carleson measure but does not satisfy condition (Ch).

On the other hand, define the sequences $s_{i}:=\frac{1}{3^{i}}-1$ and $b_{i}:=\frac{1}{2^{i}}$. Then the measure $\sigma=\sum_{i=1}^{\infty} b_{i} \delta_{s_{i}}$ is finite.

Now, consider for each non-negative integer $k$ the interval $I_{k} \subset \partial \mathbb{D}$ centered at -1 and with length $\left|I_{k}\right|=\frac{1}{3^{k}}$. Then

$$
\begin{aligned}
\int_{S\left(I_{k}\right)}|1-z|^{2} d \sigma(z) & =\sum_{r_{i}<\left|I_{k}\right|-1}\left(1-s_{i}\right)^{2} b_{i} \\
& \gtrsim \sum_{i=k+1}^{\infty} \frac{1}{2^{i}}=\frac{1}{2^{k}} .
\end{aligned}
$$

Thus, $\frac{\int_{S\left(I_{k}\right)}|1-z|^{2} d \sigma(z)}{\left|I_{k}\right|} \geq\left(\frac{3}{2}\right)^{k} \rightarrow \infty$ when $k$ tends to infinity. Hence $\sigma$ is not a $D\left(\delta_{1}\right)$-Carleson measure. However, $\sigma$ satisfies condition $(\mathrm{Ch})$ :

$$
\int \frac{1-|z|^{2}}{|1-z|^{2}} d \sigma(z) \lesssim \sum_{i=1}^{\infty} \frac{1}{2^{i}}<\infty
$$

\section{ACKNOWLEDGMENT}

This work appeared as part of the author's doctoral dissertation at the University of Tennessee under the supervision of Dr. Stefan Richter. The author thanks Dr. Richter for his help and encouragement during the development of this work.

\section{REFERENCES}

[1] J. Agler, A disconjugacy theorem for Toeplitz operators, Amer. J. Math. 112 (1990), 1-14. MR.1037599 (91b:47048)

[2] A. Aleman, The Multiplication Operators on Hilbert Spaces of Analytic Functions, Habilitationsschrift, Fernuniversität Hagen (1993).

[3] R. Chartrand, Toeplitz operators on Dirichlet-type spaces, J. Oper. Theory 48 (2002), 3-13. MR.1926041 (2003g:47046)

[4] R. Chartrand, Multipliers and Carleson measures for $D(\mu)$, Integr. Eq. Oper. Theory 45 (2003), 309-318. MR1965337 (2004b:30088)

[5] P. Duren, Theory of $H^{p}$ Spaces, Dover Publications (2000)

[6] S. Richter, A representation theorem for cyclic analytic two-isometries, Trans. Amer. Math. Soc. 328 (1991), 325-349. MR 1013337 (92e:47052)

[7] S. Richter, C. Sundberg, A formula for the local Dirichlet integral, Michigan Math. J. 38 (1991), 355-379. MR1116495 (92i:47035)

[8] S. Richter, C. Sundberg, Multipliers and invariant subspaces in the Dirichlet space, J. of Operator Theory 28 (1992), 167-187. MR.1259923 (95e:47007) 
[9] W. Rudin, Real and Complex Analysis, McGraw-Hill (1987). MR924157 (88k:00002)

[10] D. Sarason, Local Dirichlet spaces as de Branges-Rovnyak spaces, Proc. Amer. Math. Soc. 125 (1997), 2133-2139. MR1396993 (98h:46023)

[11] D. Sarason, Harmonically weighted Dirichlet spaces associated with finitely atomic measures, lntegr. Equ. Oper. Theory 31 (1998), 186-213. MR1623461 (99i:46015)

[12] A. Serra, Interpolating sequences in harmonically weighted Dirichlet spaces, Proc. Amer. Math. Soc. 131 (2003), 2809-2817. MR1974338(2004b:30069)

[13] S. Shimorin, Complete Nevanlinna-Pick property of Dirichlet type spaces, J. Func. Anal. 191 (2002), 276-296. MR1911187 (2003c:47032)

[14] S. Shimorin, Reproducing kernels and extremal functions in Dirichlet-type spaces, J. Math. Sci. 107 (2001), 4108-4124. MR.1692900 (2000g:46034)

[15] D. Stegenga, Multipliers of the Dirichlet space, Illinois J. Math. 24 (1980), no. 1, 113-139. MR.550655 (81a:30027)

[16] Z. Wu, Carleson measures and multipliers for Dirichlet spaces, J. Funct. Anal. 169 (1999), 148-163. MR 1726750 (2000k:30081)

[17] K. Zhu, Operator Theory in Function Spaces, Mathematical Surveys and Monographs, American Mathematical Society (2007). MR2311536 (2008i:47064)

Departamento de Matemáticas, Pontificia Universidad Javeriana, Bogotá, Colombia

E-mail address: chacong@javeriana.edu.co 